\title{
Percepción del espacio público: pérdida de atractivo en el Corredor Urbano Comercial Avenida de los Héroes de Chetumal, Quintana Roo, México
}

\author{
Agustín Murillo Pantî ${ }^{1}$ \\ ORCID: https://orcid.org/0000-0002-6834-7372 \\ Correo electrónico: agustin.murillo@uqroo.edu.mx \\ Miguel Ángel Barrera Rojas ${ }^{1}$ \\ ORCID: https://orcid.org/0000-0002-9240-7646 \\ Bonnie Lucía Campos Cámara ${ }^{1}$ \\ ORCID: https://orcid.org/0000-0003-0863-1774 \\ ${ }^{1}$ Universidad de Quintana Roo, Ciudad Chetumal, Quintana Roo, México.
}

\section{Resumen}

El artículo se centra en la percepción de la pérdida de atractivo del Corredor Urbano Comercial de la Avenida de los Héroes de la ciudad de Chetumal (CUCAH), Quintana Roo, México, como caso referente de los cambios y transformaciones que actualmente están ocurriendo a nivel global, derivando en cambios de comportamiento y uso de la ciudanía en el espacio público. Específicamente, el estudio se enfoca en la perspectiva del usuario-consumidor, a través del análisis de los atributos del Marco de las Cuatro Aes, que representa el modelo de gestión para el éxito de las Áreas Comerciales Urbanas o Centros Comerciales Abiertos (CCE), basado en la Accesibilidad, Atracciones, Amenidades y Acción; dentro del contexto de abandono y deterioro físico. El objetivo principal es conocer la opinión del ciudadano sobre los elementos de mayor incidencia en la pérdida del atractivo del corredor, que permita la generación de un documento base de orientación hacia las políticas públicas enfocadas a la renovación de espacios urbanos comerciales para la ciudad de Chetumal. Se aplica una encuesta estructurada y el análisis por medio del método descriptivo bivariado. Se concluye que el $70 \%$ de los encuestados opinan estar de acuerdo en la pérdida de atractivo del corredor. Asimismo, esta tendencia fue respaldada por los resultados de las variables independientes analizadas con Marco de las Cuatro Aes.

Palabras clave

Atractivo comercial, corredor urbano comercial, espacio público, percepción ciudadana

\section{Perception of public space: Loss of attractiveness in the Urban Commercial Corridor Avenida de los Héroes de Chetumal, Quintana Roo, Mexico}

\begin{abstract}
The article focuses on the perception of the loss of attractiveness of the Urban Commercial Corridor of Avenue of the Heroes in the city of Chetumal (CUCAH), Quintana Roo, Mexico, as a reference case of the changes and transformations that are currently taking place at a global level, resulting in changes in behavior and use of citizens in public space; the study focuses on the user-consumer perspective, through the analysis of the attributes of the $4 \mathrm{As}$, which represents the management model for the success of Town Centre Management, based on the study of Accessibility, Attractions, Amenities and Action; within the context of abandonment and physical deterioration. The main objective is to know the opinion of the citizen on the elements with the greatest incidence in the loss of the attractiveness of the corridor, which allows the generation of a base document for orientation towards public policies focused on the renovation of commercial urban spaces for the city of Chetumal. The source of information is the structured survey and analysis using the bivariate descriptive method. It has concluded that $70 \%$ of the respondents think they agree on the loss of attractiveness of the corridor. Likewise, this trend was supported by the results of the independent variables analyzed.
\end{abstract}

\section{Keywords}

Citizen perception, commercial urban corridor, public space, Town Centre Management 


\section{Introducción}

Este estudio tiene por objetivo conocer la percepción del ciudadano sobre los elementos de mayor incidencia en la pérdida del atractivo del Corredor Urbano Comercial de la Avenida de los Héroes (CUCAH). El interés de realizar esta investigación científica parte del hecho que en la literatura académica se señala que a nivel mundial se vienen experimentando cambios y transformaciones como consecuencia de los efectos generados por fenómenos económicos, sociales, culturales y tecnológicos, los cuales producen alteraciones en el comportamiento y uso de las personas en el espacio público (Segovia, 2007; Cao \& Kang, 2019; Moravcová, Jiri, Pekna, Moravcova \& Novakova, 2020), de los cuales, muchos derivan en el efecto de abandono y pérdida de la centralidad. En este contexto, la pregunta de investigación que guía el desarrollo de este trabajo es ¿Qué origina la pérdida del atractivo del CUCAH?, refiriéndose no únicamente a la dimensión físico-edificada, sino a todos los atributos que hacen de un espacio público urbano comercial sea exitoso.

Este texto es la primera parte que dará cuenta del contexto histórico, social y económico del CUCAH en Chetumal, Quintana Roo, México. Posteriormente, se discute en el apartado conceptual la relación que existe de la percepción ciudadana del espacio público en el marco de las relaciones sociales que se dan entre la ciudadanía, así como el abordaje del Centro Comercial Abierto/Urbano como estrategia de atractivo comercial. La tercera sección explica la metodología del Marco de las Cuatro Aes, que agrupa la Accesibilidad, Atracciones, Amenidades y Acción, a partir de un modelo de análisis confirmatorio de orden superior, para la constatación de la incidencia de los factores (Cerdá Suárez, Fernández Nogales y Rebollo Arévalo, 2007; Cerdá Suárez, 2016), misma que fue aplicada a través de encuestas a personas que radican en diversas colonias de la ciudad de Chetumal durante el uso-consumo dentro del CUCAH. Este método reduce la certidumbre en las decisiones en los proyectos de intervención urbanístico para revertir los problemas de pérdida del atractivo en áreas comerciales urbanas. Por lo anterior, en el presente artículo se aborda el estudio de los indicadores que integran los factores del Marco de las Cuatro Aes adaptado a la ciudad de Chetumal con caso de estudio del CUCAH desde la perspectiva del ciudadano.

El corredor urbano de la Avenida de los Héroes se caracterizó desde sus inicios en el siglo pasado como el lugar de mayor importancia de diferenciación que poseía la ciudad de Chetumal y la sociedad; se trata de un espacio público abierto donde se mezclaban actividades de comercio, cultura, servicios, esparcimiento, ocio y tiempo libre. Ubicado en la parte fundacional de la ciudad (Figura 1), con una longitud de $1.250 \mathrm{~m}$, se extiende en sentido norte-sur a lo largo de 10 cuadras urbanas, comprendidas entre la calle 22 de enero (actualmente peatonalizada) en el extremo sur y la calle Cristóbal Colón en el extremo norte (H. Ayuntamiento de Othón P. Blanco, 2018).

La época de esplendor se dio a partir de la declaratoria del Estado de Quintana Roo como categoría de Zona Libre de Impuestos el 30 de junio de 1992, con una duración de ocho años; sin embargo y a pesar de la entrada del Acuerdo General sobre Aranceles Aduaneros y Comercio (GATT) en el año de 1986, la consideración de Zona Libre fue prorrogada con diversas modalidades hasta el año de 1993, entre ellas una tasa del I.V.A. del 10\% y no del 15\% como en el resto del país. Con la llegada del Tratado de Libre Comercio (TLC) que entró en vigor el 1 de enero de 1994, la situación de prosperidad comercial del corredor urbano al igual que el resto de toda la ciudad se vio perjudicada, en virtud, que el sustento de comercio era la venta de productos de importación (Xacur Maiza, 2017).

El crecimiento urbano de la ciudad de Chetumal orientado a la zona norte, trajo consigo la inserción de nuevos corredores urbanos comerciales como la Av. Erick Paolo Martínez y la Av. Maxuxac, así como el establecimiento franquicias nacionales e internacionales, nuevos desarrollos habitacionales, supermercados y la construcción de la Plaza Comercial de las Américas, que paulatinamente ha diversificado el sector económico y vino a reforzar los nuevos espacios urbanos comerciales 
Figura 1

Ubicación geográfica del Corredor Urbano Comercial de la Avenida de los Héroes.
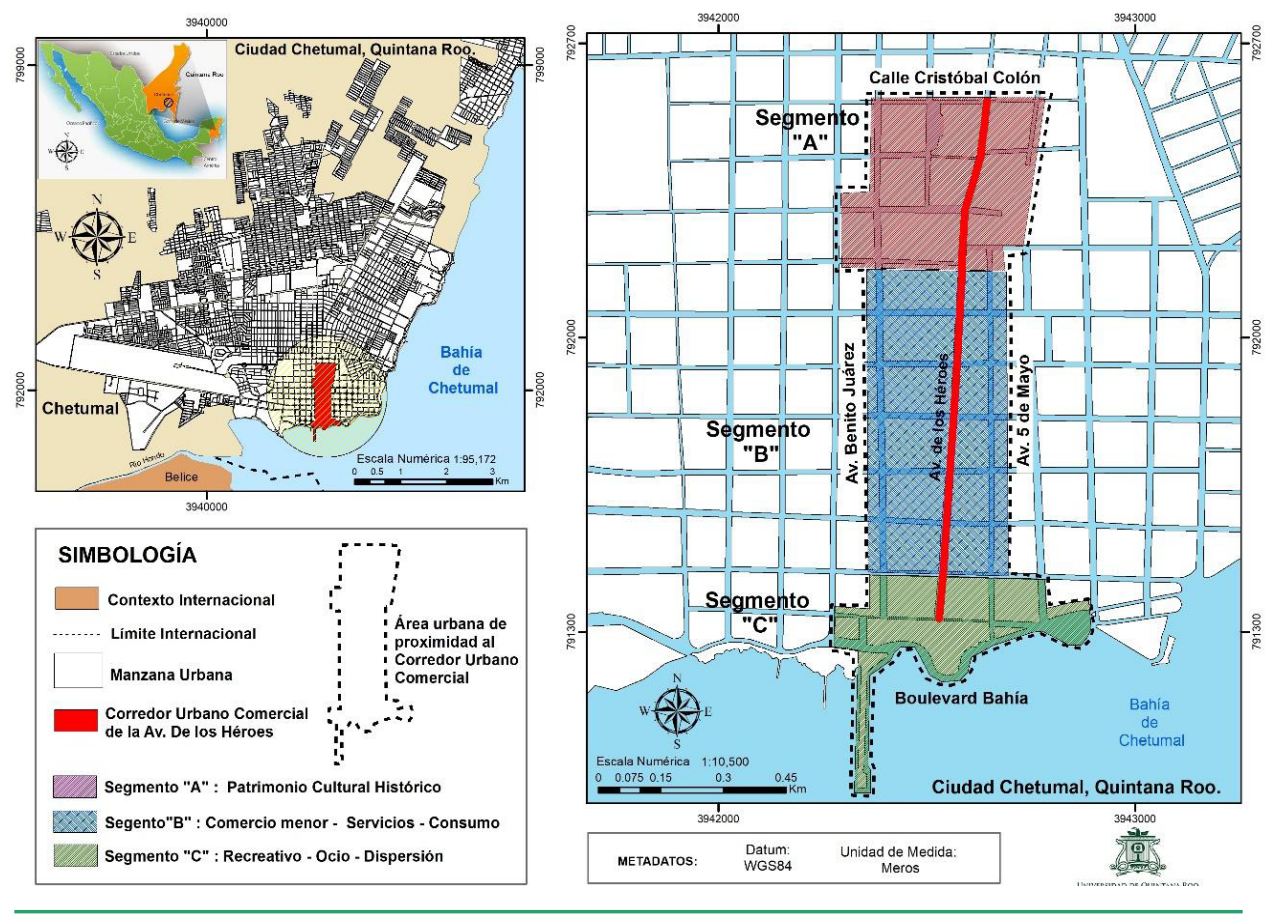

Fuente: Elaboración propia.

a partir de una nueva centralidad espacial urbana, revalorizando el uso del suelo de las colonias contiguas a estos lugares. Sin embargo, ese comportamiento de posibilidades encaminado a equilibrar el crecimiento y el desarrollo no es alentador para el corredor urbano comercial de la Avenida de los Héroes, pues hoy en día este espacio presenta un comportamiento que pareciera ser de subsistencia, porque la actividad comercial ya no es rentable para los empresarios, ni atractiva a los visitantes. Es un espacio público con alto arraigo social, pero que agoniza desde el punto de vista económico y de coyuntura espacial en la ciudad. Este espacio que por su actividad comercial y gubernamental alguna vez fue corazón y pulmón de la economía sur fronteriza, ha sido relegado a la venta de calzado, ropa y accesorios telefónicos de baja calidad, con tendencia a la piratería, que se importan desde la zona libre de Belice.

Durante el Sexenio del gobernador Roberto Borge Angulo (2011-2016) se destinó la cantidad de 110 millones de pesos mexicanos del presupuesto de egresos del ejercicio fiscal 2014 (Poder Ejecutivo, Secretaría de Hacienda y Crédito Público, 2013), contemplados en el rubro de Proyectos de Infraestructura Social, para la remodelación de la emblemática e histórica Avenida de los Héroes, dotándola de una nueva y funcional imagen para hacerla más atractiva considerándola como el núcleo comercial de la capital del Estado, a través de la construcción de $1.200 \mathrm{~m}^{2}$ de concreto hidráulico estampado, jardinería, iluminación, e instalaciones electromecánicas y de agua potable subterráneas. El día 10 de septiembre de 2016, la obra fue inaugurada con grandes expectativas de ver materializada la reactivación económica (Márquez Ulin, 2016), sin embargo, la obra no fue concluida en su totalidad, quedando algunos conceptos pendientes por ejecutar. En la actualidad y después de haber transcurrido casi cuatro años de gobierno del Gobernador C.P. Carlos Joaquín González, la problemática de decadencia del Corredor Urbano Comercial de la Avenida de los Héroes (CUCAH) aún continúa.

\section{Percepción ciudadana del espacio público}

El espacio público ha sido abordado desde diferentes enfoques disciplinarios y en función del objeto de estudio. En la literatura académica se puede hallar evidencia, por ejemplo, del análisis entre la relación del espacio público y la violencia (Aguirre y Fuentes, 2013); las formas de apropiación cultural del espacio público (Calderón, Giraldo y Jiménez, 2018); los procesos de segregación y gentrificación en y del espacio público (Bensús, 2012; García y Ruíz, 2011; Janoschka, 2016); y un buen número 
de trabajos que abordan la percepción del espacio público (Arroyo, 2018; Calderón, Gómez, Dávila, Osorio, \& Caro, 2020; López, López y Carvajal, 2020); sin embargo, son pocos los trabajos que lo hacen desde la perspectiva de la pérdida de atractivo y su desvaloración.

En sentido de lo anterior, conviene definir al espacio público; para fines de este trabajo, se entiende como espacio público a aquél que "Es un espacio de dominio público, uso social y colectivo, multifuncional, estructurador de la actividad privada y locus privilegiado de la inclusión” (Carrión, 2016 en Ramírez, 2016, p. 39). El autor refiere que el espacio público en este contexto otorga calidad a la ciudad, define su cualidad y por tal, el espacio público es el eje estratégico de la ciudad actual. La calidad conferida a la ciudad a través de los espacios públicos está sustentada por la intensidad de las relaciones sociales que se suscitan entre la ciudadanía, donde las mezclas de grupos y comportamientos toman mayor relevancia dentro de un contexto de accesibilidad constante, dando paso a la identificación del espacio (Pascual-González y Peña-Díaz, 2012). Por lo anterior, el espacio público es el lugar por excelencia de la convivencia de la ciudadanía, generadora de imágenes y experiencias de la vida cotidiana, donde:

La percepción del espacio público desde la opinión de la gente no está medida por la imposición normativa de la definición que establece la Ley, donde el concepto del interés colectivo de lo público y lo privado prima sobre lo demás, sino por la posibilidad de hacer o no uso de este espacio. (Pérez, 2004, p. 29)

En este sentido, es imperativo destacar que las percepciones no se circunscriben por las agencias de desarrollo privadas o gubernamentales, sino, por los ciudadanos que habitualmente van generando vivencias en el uso de los espacios públicos. El enfoque estético del espacio público no condiciona la percepción de la ciudadanía, en virtud, que el enfoque social es la clave que privilegia la interpretación, tal como lo plantea GarcíaDoménech (2014), sólo cuando la sociedad identifica y hace suyo el uso y función de un espacio público, comienza a preocuparse de su dimensión estética; no obstante, reconoce que la estética forma parte del espacio público, por tal, cuando el enfoque estético es bueno, la percepción de la ciudadanía hacia el espacio público aumenta. Así mismo, en la percepción ciudadana del espacio público también converge el desarrollo de las actividades comerciales, que lejos de verse como algo perjudicial, es parte del comportamiento de hacer ciudadanía, siempre y cuando exista el equilibrio, dando paso a una mayor manifestación sociocultural sobre la comercialización (García-Doménech, 2015). En este contexto, la percepción ciudadana del espacio público para el caso de estudio busca el entendimiento actual de las dinámicas de relación vinculadas al uso y consumo en el CUCAH de manera cotidiana, tanto de manera espacial, como de convivencia y disfrute del espacio público, para tal efecto, cobra relevancia la información de primera mano, proporcionada por los actores participantes de la investigación, a través de la opinión individual.

\section{Centro Comercial Abierto/Urbano como estrategia de atractivo comercial}

En las últimas décadas, se presenció una nueva forma de llevar a cabo la actividad comercial en las ciudades, que fue definiendo nuevos espacios y maneras de comercialización de diversos tipos de productos y servicios en un solo espacio, de grandes dimensiones, con ambiente confortable y todo al alcance del consumidor y recibieron el nombre de Centros Comerciales. Éstos agrupan en su interior grandes tiendas departamentales especializadas, donde las mercancías adquieren gran carga simbólica y al mismo tiempo son efímeras, generalmente ubicadas en la parte periférica de las ciudades (Gasca Zamora, 2017). Esta nueva forma de comercialización en la ciudad vino a ganar terreno a la forma tradicional del comercio urbano, principalmente el ubicado en los centros históricos o tradicionales, generalmente montado en una pequeña superficie de venta y de propiedad de un solo dueño; con la poca o nula capacidad de competencia ante nuevos esquemas modernos de planificación, operación y comercialización de productos de todo el mundo (García Gallego, Chamorro Mera y Palacios González, 2010). Aunado a lo anterior, el comercio urbano tradicional sufrió 
un fuerte embate ante las nuevas reglas de la liberación y globalización de los mercados a nivel mundial, con nuevos requerimientos tecnológicos y aplicaciones informáticas digitales, a los cuales no estaban familiarizados, haciendo que muchos comerciantes sucumbieran ante la falta de incorporación a esta nueva dinámica de competencia comercial (Flórez Parra, 2011). Estos centros comerciales cerrados encontraron una fortísima competencia y para el consumidor resultó una alternativa, la conformación de Centros Comerciales Abiertos (CCA) (Iso Tinoco, 2013; Vecslir y Rodríguez, 2018), también conocidos como Centros Comerciales Urbanos, dependiendo de la región geográfica donde se localicen.

Estos CCA, en el contexto de financiamiento de proyectos viables de desarrollo económico y social para América Latina y el Caribe por el Banco Interamericano de Desarrollo (BID), representaron en la década de 1990 una opción de política urbana bien recibida por los grandes capitales latinoamericanos, puesto que los gobiernos locales comenzaban a verse rebasados por la distorsión de precios en el suelo en la periferia urbana que ocasionaban los grandes centros comerciales, y por el comercio ilegal en la vía pública (Vecslir y Rodríguez, 2018). Desde luego, México se sumó a esta dinámica para fortalecer el atractivo comercial y recuperación de la centralidad espacial.

\section{Un CCA es definido como}

Una agrupación o aglomeración espacial y permanente de un número indeterminado de establecimientos comerciales minoristas, independientes entre sí y correspondiendo a diferentes sectores (comercios especialistas o generalistas, pero con servicios diversos y actividades de restauración, ocio e incluso culturales y turísticas), situados juntos o en estrecha proximidad, pero no en un solo edificio, sin ser el resultado de una operación planificada previa del conjunto sino más bien fruto del desarrollo de los intereses individuales de los comerciantes, ubicados en las tramas urbanas de pueblos y ciudades, y con una gestión unitaria que les proporciona una imagen externa común (Cerdá Suárez, 2006 en García et al., 2010, p. 1-2).
Asimismo, este esquema de CCA debe propiciar un ambiente y espacio atractivo para el visitante, por ello debe ajustarse a las medidas de accesibilidad y movilidad, estacionamientos, oferta comercial, entorno urbano, imagen común, seguridad, control del clima, gestión conjunta, y servicios y actividades complementarias (S.I.C.I. DOMINUS.SL., 2007).

Por anterior y, de acuerdo con Pancorbo Sandoval, Benavides y León Sánchez (2011), los CCA son generadores de una gran vitalidad, dinamismo y rentabilidad entre la ciudad y éste. Por tal, el CUCAH se aborda desde este enfoque motivado por su conformación físico-espacial, su vinculación con la ciudad y el reconocimiento ciudadano como lugar tradicional de comercio.

\section{Materiales y Métodos}

El enfoque metodológico empleado en la investigación es cuantitativa descriptiva, destacando aspectos relevantes del espacio público urbano a través de la percepción del ciudadano en su rol de usuario-consumidor inherente al atractivo de la zona comercial abierta. Geográficamente, el estudio contempla a toda la población de la ciudad de Chetumal que acude al CUCAH, con la expectativa de contar con una muestra representativa de todas las clases socioeconómicas, con diversidad de preferencias, gustos, comportamientos de compras y ocio diferenciados. La información se obtiene directamente en campo, a través de la aplicación de una encuesta diseñada y adaptada al caso de estudio, con una variable dependiente directamente relacionada con la pérdida del atractivo del CUCAH y cuatro variables independientes vinculadas a los atributos de éxito de espacios urbanos comerciales; los resultados obtenidos son transferidos a datos numéricos mediante instrumentos estadísticos.

A partir de la literatura del Marco de las Cuatro Aes, Cerdá Suárez et al. (2007) establecen una propuesta de identificación y evaluación de estrategias para abordar temas de revitalización de C.C.A., proponiendo un listado de recursos y capacidades para el éxito de estos espacios urbanos. Esta propuesta plantea cuatro dimensiones: Accesibilidad, Atracciones, Amenidades y 
Tabla 1

Características centrales de los participantes de la investigación

Variable Dependiente
¿La zona comercial de la Av. de los Héroes ha perdido su atractivo?

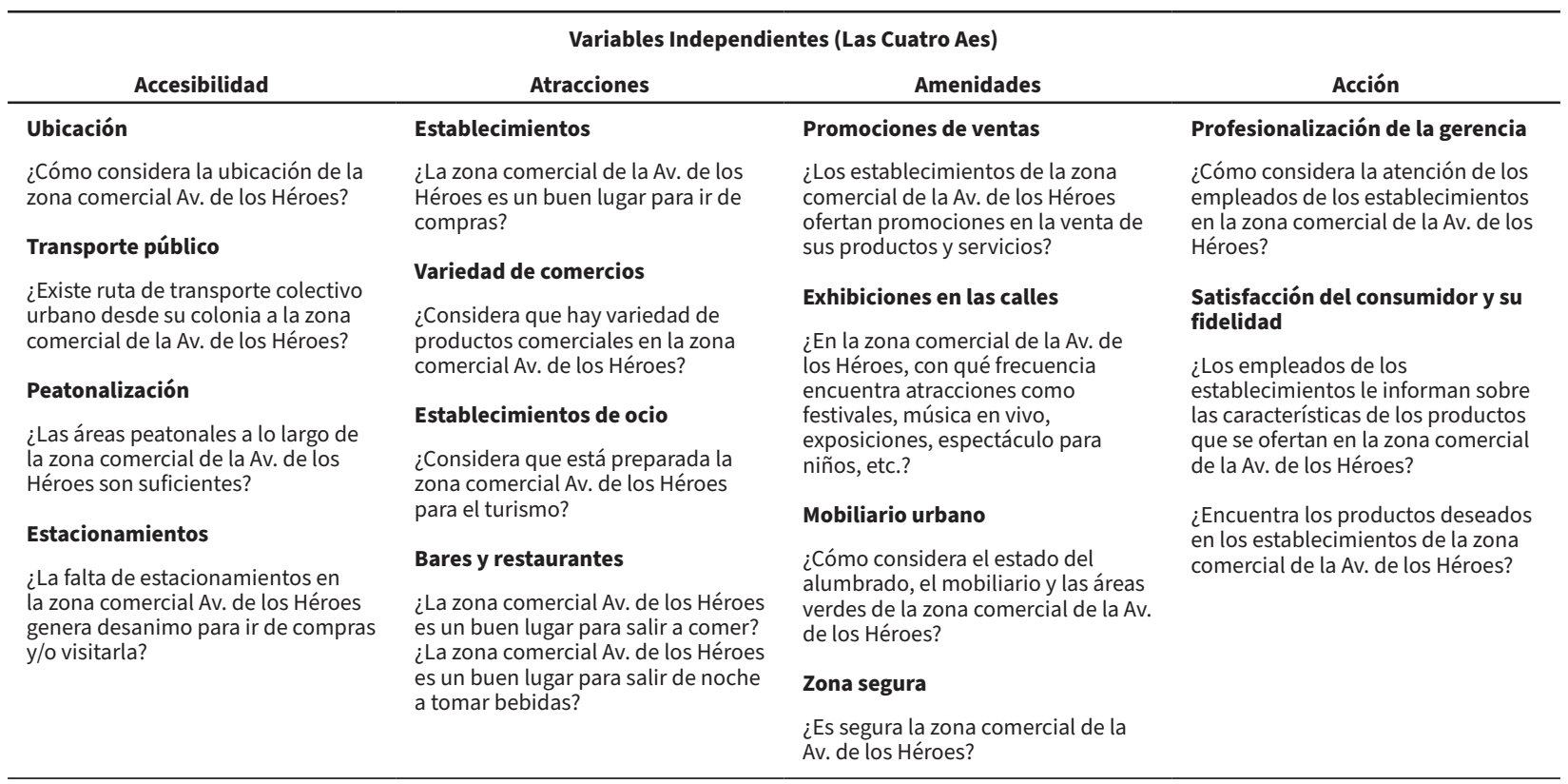

Fuente: Elaboración propia, con base en el Marco de las Cuatro Aes y Atributos de Cerdá Suárez et al. (2007) y Cerdá Súarez (2016).

Acción. La Accesibilidad, es referida como un elemento indispensable de ubicación de las zonas comerciales dentro de la ciudad. La Atracción, vista desde el enfoque de marketing, como herramienta indispensable en el siglo XXI para obtener el mayor beneficio del comportamiento del consumidor. Las Amenidades, indispensables para fortalecer la atracción del área comercial, a través de elementos con los cuales las personas se puedan identificar. En el plano de lo no tangible se contemplan las promociones en el proceso de comercialización; y en el espacio común la incidencia de actividades lúdicas que hagan del lugar una vivencia distinta en la visita. Por último, la Acción, encaminada a la capacidad de gestión por parte del comerciante, así como a las promociones como estrategia de satisfacción. La suma de las cuatro dimensiones permite alcanzar la ventaja competitiva comercial encaminada al éxito.

Con base a los indicadores establecidos en Cerdá Suárez et al. (2007) y Cerdá Suárez (2016), se estructuró la siguiente tabla Marco de las Cuatro Aes con aplicación al CUCAH de la ciudad de Chetumal (Tabla 1), destacando que en la actualidad es necesario y de suma importancia estudiar las necesidades y percepciones que tienen los clientes de una zona comercial para el desarrollo de estrategias espaciales y de comercialización que ayuden a mejorar el atractivo de un espacio como lo es el CUCAH.

Con la finalidad de conocer los elementos que actúan sobre la pérdida del CUCAH desde la perspectiva del usuario-consumidor, el proceso metodológico empleado en la investigación retoma el análisis empírico del Marco de las Cuatro Aes con el enfoque establecido por (Cerdá Suárez et al., 2007), en el contexto de aplicación a Zonas Comerciales Urbanas y/o Centros comerciales Abiertos. La identificación y adaptación de los indicadores que conforman las cuatro dimensiones de las Aes derivaron de la revisión de literatura, observación de campo y aplicación de encuestas de pilotaje del día 13 de marzo de 2020. Los actores participantes en el desarrollo de la investigación se rigieron bajo los siguientes criterios: Usuarios-consumidores residentes de las colonias de la ciudad de Chetumal; el perfil del actor se estratificó en dos grupos, Mujeres y Hombres, con porcentajes igualitarios (H 50\% - M 50\%); la edad mínima del actor participante fue a partir de los 18 años. Para la determinación de la muestra se consideró la población total de la ciudad de Chetumal, la cual es de 151.243 habitantes de acuerdo al Censo de Población y Vivienda del año 2010 (Instituto Nacional de Estadística y Geografía, 2010), y de acuerdo al 
cálculo realizado por medio de la fórmula para poblaciones grandes, se determinó la cantidad de 100 encuestas por aplicar, con un nivel de la confianza del $90 \%$, margen de error máximo admitido del 7\% y desviación estándar del 0,42 (obtenido del pilotaje). Las encuestas fueron programadas para su aplicación en fechas 19, 20, 21, de marzo, siendo los días jueves, viernes y sábado respectivamente, correspondiendo a los días de mayor concurrencia de visita, uso y consumo de la ciudadanía en el CUCAH. Las encuestas se diseñaron con reactivos cuya opción de respuesta se diera en escala de Likert, con aplicación en sitio. El método descriptivo bivariado fue empleado para el análisis de los resultados de la variable dependiente y las independientes.

\section{Resultados}

Las etapas de análisis de resultados se realizaron en dos secciones. Primeramente, se realizó la valoración de los resultados de la variable dependiente, relativa a la percepción que tienen los usuarios-consumidores de la pérdida de atractivo del CUCAH, y posteriormente se analizaron los resultados de las variables independientes, conformadas a partir del Marco de las cuatro Aes. Para el primer caso, el análisis probabilístico se realizó con el total de habitantes encuestados, y seguidamente agrupando las percepciones por rangos de género, en condiciones igualitarias de número. Los resultados obtenidos son los siguientes (Tabla 2):

Tabla 2

Resultados de la variable dependiente del CUCAH (población total encuestada y encuestados por género)

\begin{tabular}{|c|c|c|c|c|}
\hline \multirow[b]{2}{*}{ Percepción } & \multicolumn{3}{|c|}{$\begin{array}{l}\text { Pérdida de atractivo del Corredor Urbano Comercial de la Av. de los Héroes } \\
\text { (Población total encuestada) }\end{array}$} & \multirow[b]{2}{*}{ Pmd. Ponderado } \\
\hline & Encuestados & Valor & Ponderación & \\
\hline Totalmente de acuerdo & 32 & 5 & 0,32 & 1,6 \\
\hline De acuerdo & 38 & 4 & 0,38 & 1,52 \\
\hline Indeciso & 14 & 3 & 0,14 & 0,42 \\
\hline En desacuerdo & 14 & 2 & 0,14 & 0,28 \\
\hline Totalmente en desacuerdo & 2 & 1 & 0,02 & 0,02 \\
\hline
\end{tabular}

Pérdida de atractivo del Corredor Urbano Comercial de la Av. de los Héroes

(Encuestados género masculino)

\begin{tabular}{lllll} 
Percepción & Encuestados & Valor & Ponderación & Pmd. Ponderado \\
\hline Totalmente de acuerdo & 18 & 5 & 0,36 & 0,32 \\
\hline De acuerdo & 16 & 4 & 0,16 & 1,28 \\
\hline Indeciso & 8 & 3 & 0,16 & 0,48 \\
\hline En desacuerdo & 8 & 2 & 0 & 0 \\
\hline Totalmente en desacuerdo & 0 & 1 & 0,32 \\
\hline
\end{tabular}

Pérdida de atractivo del Corredor Urbano Comercial de la Av. de los Héroes

(Encuestados género femenino)

\begin{tabular}{lllll} 
Percepción & Encuestados & Valor & Ponderación & Pmd. Ponderado \\
\hline Totalmente de acuerdo & 14 & 5 & 0,28 & 1,4 \\
\hline De acuerdo & 22 & 4 & 0,44 & 1,76 \\
\hline Indeciso & 6 & 3 & 0,12 & 0,36 \\
\hline En desacuerdo & 6 & 2 & 0,12 & 0,24 \\
\hline Totalmente en desacuerdo & 2 & 1 & 0,04 & 0,04 \\
\hline
\end{tabular}

Fuente: Elaboración propia. 
La variable dependiente fue planteada con cinco opciones diferentes para recabar la opinión de la población, destacando que el 70\% seleccionaron las alternativas afirmativas de la pérdida del atractivo, es decir, el $38 \%$ está de acuerdo y el 32\% totalmente de acuerdo; mientras que $14 \%$ opinó estar en desacuerdo y el $2 \%$ en totalmente en desacuerdo; el 14\% de los encuestados se mantuvieron indecisos. De lo anterior, se puede decir en términos generales que la variable dependiente fue calificada con un amplio margen como pérdida de atractivo del CUCAH. El factor edad de los usuariosconsumidores se vio directamente vinculada en la percepción de la variable dependiente, en promedio a mayor edad (>30 años), mayor afinidad con las respuestas de opciones afirmativas. Lo anterior, se puede explicar que las personas con mayor edad tienen la posibilidad de comparación del actual corredor urbano comercial con el vivido en años pasados, principalmente cuando estaba instaurada la zona franca libre de impuestos o más próximo a la etapa de comercialización con un impuesto del 10\% de I.V.A. Evidentemente, para ese entonces la actividad comercial en el corredor urbano era de mayor dinamismo comercial, asimismo, ese espacio público era el referente comercial local de toda la ciudad de Chetumal. Por el contrario, las personas más jóvenes (<30 años) encuestadas difícilmente pueden tener la consideración de la experiencia vivida en el lugar para realizar el comparativo. No obstante, existen otros indicadores que no necesariamente se vinculan con la edad para determinar a través de la percepción que el corredor urbano comercial ha perdido su atractivo, los cuales se presenta más adelante.

En cuanto a la percepción de la variable dependiente por género de los encuestados, la población femenina presentó un marcado distanciamiento con un $12 \%$ de diferencia en la opción afirmativa de acuerdo en relación con la población masculina; y en la opción totalmente de acuerdo fue lo contrario, aunque con un margen de diferencia menor (8\%); y se mantuvo un $4 \%$ de margen para las dos opciones siguientes (indeciso y en desacuerdo), en ambos casos con inclinación al género masculino (Tabla 1).
Como hemos visto, tanto hombres como mujeres afirman que la pérdida del atractivo del CUCAH ha ocurrido, situación por demás preocupante toda vez que se trata de la percepción del usuario y consumidor que hace la diferenciación al elegir un espacio comercial urbano abierto a través de su preferencia.

Los resultados del análisis en el contexto del Marco de las cuatro Aes con adaptación local se describen a continuación, iniciando primeramente con los indicadores que agrupan la dimensión de la Accesibilidad del CUCAH (Tabla 3).

Los indicadores de la dimensión de Accesibilidad en el espacio público abierto representan la puerta de acceso para el estudio de la viabilidad del atractivo comercial, el lugar debe ser accesible para todos los usuarios y consumidores, tanto espacialmente como en la parte no tangible que involucra el marketing; garantizar la movilidad de las personas desde la colonia de procedencia a través de los diferentes medios de transporte, tanto públicos como privados, privilegiando en todo momento el acceso peatonal (García Gallego et al., 2010). Al respecto, de los resultados obtenidos en el indicador relativo a la evaluación de la ubicación del CUCAH, el 84\% coincidieron que es buena, muy buena y excelente, siendo la opción buena como la más elegida con un $46 \%$ de toda la población encuestada. El comportamiento en la selección de las respuestas según el género es muy similar. La base de que los usuariosconsumidores hayan optado con un amplio margen por la confirmatoria de buen lugar para el corredor, en gran medida se respalda a su localización dentro del Centro Histórico y Centro Urbano de la ciudad de Chetumal, lugar donde se asientan las edificaciones representativas y de historia que han forjado la identidad del Chetumaleño. Asimismo, las vías de comunicación al interior de la ciudad que confluyen al corredor son de trazo ortogonal de fácil lectura urbana y de dimensiones amplias.

Dentro de los hallazgos del indicador de transporte colectivo urbano desde la colonia de procedencia de los visitantes al corredor, destaca que el $50 \%$ de los encuestados refirieron no contar con este medio de transporte; 
Tabla 3

Resultados de la dimensión de Accesibilidad en el Marco de las Cuatro Aes del CUCAH

\begin{tabular}{|c|c|c|c|c|}
\hline \multirow[b]{2}{*}{ Percepción } & \multicolumn{3}{|c|}{$\begin{array}{c}\text { Accesibilidad } \\
\text { ¿Cómo considera la ubicación del Corredor Urbano Comercial de la Av. De los Héroes? }\end{array}$} & \multirow[b]{2}{*}{ Pmd. Ponderado } \\
\hline & Encuestados & Valor & Ponderación & \\
\hline Excelente & 16 & 5 & 0,16 & 0,8 \\
\hline Muy buena & 22 & 4 & 0,22 & 0,88 \\
\hline Buena & 46 & 3 & 0,46 & 1,38 \\
\hline Indiferente & 14 & 2 & 0,14 & 0,28 \\
\hline Totalmente en desacuerdo & 2 & 1 & 0,02 & 0,02 \\
\hline
\end{tabular}

¿Existe ruta de transporte colectivo urbano desde su colonia al Corredor Urbano Comercial de la Av. de los Héroes?

\begin{tabular}{lllll} 
Percepción & Encuestados & Valor & Ponderación & Pmd. Ponderado \\
\hline SI & 50 & 1 & 0,5 & 0,5 \\
\hline NO & 50 & 0 & 0,5 & 0 \\
\hline
\end{tabular}

¿Las áreas peatonales a lo largo del Corredor Urbano Comercial de la Av. de los Héroes son suficientes?

\begin{tabular}{lllll} 
Percepción & Encuestados & Valor & Ponderación & Pmd. Ponderado \\
\hline Totalmente suficientes & 0 & 5 & 0 & 0 \\
\hline Suficientes & 54 & 4 & 0,54 & 0,04 \\
\hline Indeciso & 4 & 3 & 0,16 & 0,12 \\
\hline Insuficientes & 40 & 2 & 0,02 & 0,02 \\
\hline Totalmente insuficientes & 2 & 1 & 0,0 & 0 \\
\hline
\end{tabular}

¿La falta de estacionamientos en el Corredor Urbano Comercial de la Av. de los Héroes genera desanimo para ir de compras y/o visitarlo?

\begin{tabular}{lllll} 
Percepción & Encuestados & Valor & Ponderación & Pmd. Ponderado \\
\hline Totalmente de acuerdo & 22 & 5 & 0,22 & 1,1 \\
\hline De acuerdo & 42 & 4 & 0,42 & 0,24 \\
\hline Indeciso & 24 & 3 & 0,68 & 0,72 \\
\hline En desacuerdo & 8 & 2 & 0,16 \\
\hline Totalmente en desacuerdo & 4 & 1 & 0,04 & 0,04 \\
\hline
\end{tabular}

Fuente: Elaboración propia.

llamando la atención que las mujeres externalizaron con un $32 \%$ la falta del transporte colectivo urbano, situación preocupante, ya que esto es un indicativo de desánimo para visitar el corredor urbano comercial. Tal situación no es responsabilidad de los comerciantes establecidos en el corredor, sino de la autoridad municipal local. Las áreas peatonales existentes desde la percepción del usuario en términos generales están agrupadas en dos opciones recurrentes, un 54\% las considera como suficientes y $40 \%$ insuficientes. Lo cierto es, que, hablando en términos espaciales, actualmente el porcentaje mayor de superficie de área pública de tránsito está destinada al vehículo y no al peatón. La falta de estacionamientos, ya sean públicos o privados, fue calificada de manera agrupada con un 64\% las opciones confirmatorias.
En resumen, los 4 indicadores evaluados de la Accesibilidad señalan que la ubicación y las áreas peatonales son percibidas de manera positiva; el transporte colectivo urbano se sitúa en una posición media. No obstante, desde la perspectiva del Marco de las cuatro Aes se considera como situación de desánimo para la accesibilidad; y por último, con una marcada percepción negativa se encuentra la falta de estacionamientos públicos en el CUCAH.

En lo referente a la dimensión de Atracciones, considerada como la parte que genera los vínculos de preferencia tanto por los elementos físicos, versatilidad del espacio público y variedad de marcas y productos, derivado del análisis estadístico de las respuestas de la población encuestada, se tiene los siguientes resultados (Tabla 4): 
Tabla 4

Resultados de la dimensión de Atracciones en el Marco de las Cuatro Aes del CUCAH

Atracciones

¿El Corredor Urbano Comercial de la Av. De los Héroes es un buen lugar para ir de compras?

\begin{tabular}{lllll} 
Percepción & Encuestados & Valor & Ponderación & Pmd. Ponderado \\
\hline Excelente & 8 & 5 & 0,08 & 0,4 \\
\hline Muy bueno & 16 & 4 & 0,16 & 0,64 \\
\hline Bueno & 64 & 3 & 0,64 & 0,06 \\
\hline Indiferente & 6 & 2 & 0,06 & 0,12 \\
\hline Malo & 6 & 1 & 0,06 \\
\hline
\end{tabular}

¿Considera que hay variedad de productos en el Corredor Urbano Comercial de la Av. de los Héroes?

\begin{tabular}{lllll} 
Percepción & Encuestados & Valor & Ponderación & Pmd. Ponderado \\
\hline Muy frecuentemente & 6 & 5 & 0,06 & 0,3 \\
\hline Frecuente & 64 & 4 & 0,64 & 2,56 \\
\hline Ocasionalmente & 12 & 3 & 0,12 & 0,16 \\
\hline Raramente & 16 & 2 & 0,36 & 0,32 \\
\hline Nunca & 2 & 1 & 0,02 \\
\hline
\end{tabular}

¿Considera que está preparado el Corredor Urbano Comercial de la Av. de los Héroes para el turismo?

\begin{tabular}{|c|c|c|c|c|}
\hline Percepción & Encuestados & Valor & Ponderación & Pmd. Ponderado \\
\hline Totalmente de acuerdo & 0 & 5 & 0 & 0 \\
\hline De acuerdo & 34 & 4 & 0,34 & 1,36 \\
\hline Indeciso & 18 & 3 & 0,18 & 0,54 \\
\hline En desacuerdo & 30 & 2 & 0,3 & 0,6 \\
\hline Totalmente en desacuerdo & 18 & 1 & 0,18 & 0,18 \\
\hline
\end{tabular}

¿El Corredor Urbano Comercial de la Av. de los Héroes es un buen lugar para salir a comer?

\begin{tabular}{lllll} 
Percepción & Encuestados & Valor & Ponderación & Pmd. Ponderado \\
\hline Excelente & 6 & 5 & 0,06 & 0,3 \\
\hline Muy bueno & 4 & 4 & 0,04 & 0,16 \\
\hline Bueno & 40 & 3 & 0,4 & 0,14 \\
\hline Indiferente & 14 & 2 & 0,28 & 0,36 \\
\hline Malo & 36 & 1 & 0,36 \\
\hline
\end{tabular}

¿El Corredor Urbano Comercial de la Av. de los Héroes es un buen lugar para salir de noche a tomar bebidas?

\begin{tabular}{lllll} 
Percepción & Encuestados & Valor & Ponderación & Pmd. Ponderado \\
\hline Excelente & 2 & 5 & 0,02 & 0,1 \\
\hline Muy bueno & 4 & 4 & 0,04 & 0,16 \\
\hline Bueno & 18 & 3 & 0,18 & 0,54 \\
\hline Indiferente & 26 & 2 & 0,26 & 0,52 \\
\hline Malo & 50 & 1 & 0,5 & 0,5 \\
\hline
\end{tabular}

Fuente: Elaboración propia.

El corredor fue percibido con un amplio margen (64\%) como un buen lugar para ir de compras, resultado un tanto sorprendente, si se recuerda que la variable dependiente fue ampliamente percibida como pérdida del atractivo comercial. Desde luego, tiene muchas interpretaciones, si se parte del hecho que físicamente el lugar está bien ubicado (apartado anterior). El indicador de variedad de productos fue calificado con un $64 \%$ con la opción de frecuentemente, al igual que el indicador anterior, generalmente se esperaría encontrar percepciones 
contrarias. Sin embargo, el factor socioeconómico entra en juego, como se presenta más adelante en las colonias de procedencia de los encuestados; a menor poder adquisitivo, menor variedad de productos solicitados para comprar. Lo cual, hace que las percepciones resultantes no están fuera de lugar.

De acuerdo a lo externado por los encuestados, el corredor urbano comercial no está preparado como lugar para el turismo, así lo refirieron con un $48 \%$ contra un $34 \%$ que opina que sí lo está; desde luego, el sentir ciudadano se puede palpar en los recorridos, al no encontrar establecimientos afines para propiciar la actividad turística. En la evaluación del corredor como buen lugar para salir a comer, el 50\% de los encuestados optaron por respuestas afirmativas (bueno, muy bueno, excelente), por lo que dista mucho de una confirmatoria generalizada. Es entendible tal percepción, ya que en el corredor solo se pueden encontrar 2 ó 3 lugares con amplia variedad de platillos con clima confortable, siendo lo común los establecimientos de comida rápida de venta en la banqueta o para llevar. Asimismo, el corredor calificado como buen lugar para salir de noche a tomar bebidas, el 50\% de los encuestados optaron por la opción de malo. Sorprendentemente, se vio reflejado con un mayor margen la percepción de las mujeres con esta opción, es decir, con un 10\% más que los hombres.

En resumen, de los cinco indicadores que integran la dimensión de Atracciones, sólo el primero, segundo y cuarto (buen lugar para ir de compras, variedad de productos, buen lugar para salir a comer, respectivamente) obtuvieron valoraciones positivas. En cuanto a los indicadores como lugar preparado para el turismo y para salir de noche a tomar bebidas, fueron evaluados de manera negativa.

La dimensión de las Amenidades, es la valoración a los indicadores que hacen del espacio sea un lugar ameno, agradable, placentero, calificativos vinculados que hacen que el usuario encuentre motivos para regresar al espacio público para seguir haciendo uso y disfrute del mismo. A continuación, se presentan los resultados obtenidos en la dimensión de las Amenidades (Tabla 5).
El indicador relativo a promociones en las ventas y servicios de los establecimientos de un área comercial abierta, lo deseable es que fuera de manera frecuente o muy frecuente, ya que el marketing es un incentivo ampliamente utilizado para que el consumidor sea frecuente. Para el caso de estudio, los análisis de las encuestas determinaron en suma que las opciones frecuente y muy frecuente representan el 44\% del total de las respuestas registradas; en contraparte las opciones de ocasionalmente, raramente y nunca en suma representan el 56\% de las opciones más elegidas. Lo anterior, es indicativo que negocios de comercios y servicios ubicados sobre el corredor emplean con poca frecuencia la estrategia de la promoción para hacer atractivo el lugar. En lo relativo a las amenidades por medio de atracciones, el $68 \%$ en suma optó por las opciones de ocasionalmente, raramente y nunca, situación que llama la atención por tratarse de un corredor comercial. Sin embargo, al ser de tipo abierto, con ese resultado conlleva a pensar que entre los actores propietarios de los comercios y servicios no hay la suficiente integración y/o vinculación para llevarlos a cabo.

Los indicadores de la parte física del corredor satisfactoriamente fueron percibidos con un amplio rango de estar en condiciones de bueno (54\%). En parte, esta percepción se sustenta en que los elementos que integran la parte física evaluada son de reciente suministro, en el contexto de los trabajos de remodelación de la Av. de los Héroes. No obstante, durante los recorridos se pudo observar que, si bien es cierto, se encuentran en buenas condiciones, falta la parte de los trabajos de conservación, como lo es la pintura y limpieza. Con el mismo porcentaje del $54 \%$, al sumar las opciones poco seguro e inseguro fue evaluado el indicador de espacio seguro, con mayor observancia por parte de las mujeres.

Este indicador es considerado como factor clave en el estudio del espacio público abierto (Jasso López, 2015); en gran medida las percepciones fueron sobre la base que a lo largo de corredor no hay elementos de seguridad pública de a pie, reconociendo, que los que se pueden identificar, siempre están custodiando algún edificio gubernamental y los policías generalmente circulan al 
Tabla 5

Resultados de la dimensión de Amenidades en el Marco de las Cuatro Aes del CUCAH

Amenidades

¿Los establecimientos del Corredor Urbano Comercial de la Av. de los Héroes ofertan promociones en la venta de sus productos y servicios?

\begin{tabular}{lllll} 
Percepción & Encuestados & Valor & Ponderación & Pmd. Ponderado \\
\hline Muy frecuentemente & 8 & 5 & 0,08 & 0,4 \\
\hline Frecuente & 36 & 4 & 0,36 & 0,18 \\
\hline Ocasionalmente & 18 & 3 & 0,26 & 0,54 \\
\hline Raramente & 26 & 2 & 0,52 \\
\hline Nunca & 12 & 1 & 0,12 & 0 \\
\hline
\end{tabular}

¿En el Corredor Urbano Comercial de la Av. de los Héroes con qué frecuencia encuentra atracciones como festivales, música en vivo, exposiciones, espectáculo para niños, etc.?

\begin{tabular}{lllll} 
Percepción & Encuestados & Valor & Ponderación & Pmd. Ponderado \\
\hline Muy frecuentemente & 2 & 5 & 0,02 & 0,1 \\
\hline Frecuente & 30 & 4 & 0,3 & 0,3 \\
\hline Ocasionalmente & 30 & 3 & 0,3 & 0,9 \\
\hline Raramente & 30 & 2 & 0,0 & 0,08 \\
\hline Nunca & 8 & 1 & 0 & 0 \\
\hline
\end{tabular}

¿Cómo considera el estado del alumbrado, el mobiliario y las áreas verdes del Corredor Urbano Comercial de la Av. de los Héroes?

\begin{tabular}{lllll} 
Percepción & Encuestados & Valor & Ponderación & Pmd. Ponderado \\
\hline Excelente & 2 & 5 & 0,02 & 0,1 \\
\hline Muy bueno & 6 & 4 & 0,06 & 0,24 \\
\hline Bueno & 54 & 3 & 0,54 & 0,08 \\
\hline Indiferente & 8 & 2 & 0,16 & 0,3 \\
\hline Malo & 30 & 1 & 0,3 \\
\hline
\end{tabular}

¿Es seguro el Corredor Urbano Comercial de la Av. de los Héroes?

\begin{tabular}{lllll} 
Percepción & Encuestados & Valor & Ponderación & Pmd. Ponderado \\
\hline Muy segura & 4 & 5 & 0,04 & 0,2 \\
\hline Segura & 38 & 4 & 0,38 & 1,52 \\
\hline Poco segura & 34 & 3 & 0,34 & 0,04 \\
\hline Nada segura & 4 & 2 & 0,02 & 0,2 \\
\hline Insegura & 20 & 1 & 0,2 \\
\hline
\end{tabular}

Fuente: Elaboración propia.

interior de sus unidades vehiculares. Como se pudo observar, en esta dimensión el único indicador con valoración positiva generalizada fue el estado en que se encuentran las luminarias, mobiliario urbano y áreas verdes, por los demás se suman a los indicativos de la pérdida del atractivo.

Por último, se presentan los resultados de los análisis de la dimensión de Acción (Tabla 6), agrupados en tres indicadores, tal como se puede apreciar a continuación.

En esta dimensión interviene la parte de gestión y buen trato por parte de la gerencia y empleados de los establecimientos comerciales y de servicios establecidos en el corredor urbano comercial. En este sentido, el primer indicador evaluado fue relativo al trato recibido por parte de los empleados de los establecimientos hacia los usuarios-consumidores. Al respecto, la percepción que se tiene por parte de los encuestados hacia las personas que los atiendes, es ampliamente positiva, con un $56 \%$ calificaron el trato como bueno. Esto es un buen indicativo que las personas que laboran en los comercios del corredor son en su gran mayoría de buen trato y atención. No obstante, de este buen resultado, se les cuestionó si se les informaba sobre las características de los productos que adquirían, al respecto el 24\% expresó que frecuentemente. Sin embargo, el 48\% opinaron 
Tabla 6

Resultados de la dimensión de Acción en el Marco de las Cuatro Aes del CUCAH

Acción

¿Cómo considera la atención de los empleados de los establecimientos del Corredor Urbano Comercial de la Av. de los Héroes?

\begin{tabular}{lllll} 
Percepción & Encuestados & Valor & Ponderación & Pmd. Ponderado \\
\hline Excelente & 0 & 5 & 0 & 0 \\
\hline Muy buena & 2 & 4 & 0,02 & 0,08 \\
\hline Buena & 56 & 3 & 0,56 & 0,3 \\
\hline Indiferente & 30 & 2 & 0,12 & 0,12 \\
\hline Mala & 12 & 1 & 08 \\
\hline
\end{tabular}

\begin{tabular}{llll}
$\begin{array}{l}\text { ¿Los empleados de los establecimientos le informan sobre las características de los productos que se ofertan en el Corredor Urbano Comercial de la } \\
\text { Av. de los Héroes? }\end{array}$ & Valor & Pncuestados & Ponderación \\
\hline Percepción & 0 & 5 & 0 \\
\hline Puy frecuentemente & 24 & 4 & 0,24 \\
\hline Ocasionalmente & 48 & 3 & 0,48 \\
\hline Raramente & 22 & 2 & 0,22 \\
\hline Nunca & 6 & 1 & 0,06 \\
\hline
\end{tabular}

¿Encuentra los productos deseados en los establecimientos del Corredor Urbano Comercial de la Av. de los Héroes?

\begin{tabular}{lllll} 
Percepción & Encuestados & Valor & Ponderación & Pmd. Ponderado \\
\hline Muy frecuentemente & 4 & 5 & 0,04 & 0,2 \\
\hline Frecuente & 42 & 4 & 0,42 & 0,32 \\
\hline Ocasionalmente & 32 & 3 & 0,68 & 0,96 \\
\hline Raramente & 20 & 2 & 0,4 \\
\hline Nunca & 2 & 1 & 0,02 & 0,02 \\
\hline
\end{tabular}

Fuente: Elaboración propia.

que lo hacían ocasionalmente, y con $22 \%$ raramente; evidentemente, los empleados de los establecimientos a pesar que en su mayoría son personas de buen trato, no tienen el conocimiento y/o preparación para informar debidamente sobre las características de los productos que ofertan. Asimismo, este indicador pone en evidencia que la gerencia comercial de los establecimientos no conoce tal situación o simplemente no está capacitando a su personal para proporcionar datos técnicos de los productos hacia los clientes.

$\mathrm{Y}$, por último, en relación a los productos buscados para adquirir por parte de los usuarios en el corredor, los resultados arrojaron que el $42 \%$ respondieron con un frecuentemente y $4 \%$ con muy frecuentemente, en suma, representando un $46 \%$. Este hallazgo pudiera ser sorpresivo, toda vez que la variedad de productos ofrecidos en el corredor generalmente se concentra en la comercialización de zapatos y ropa, y en menor medida en productos de enseres domésticos, productos de belleza y cosméticos. Por tal motivo, el resultado puede ser interpretado en el sentido que las personas que acuden al CUCAH buscan adquirir productos como los ya referidos, o que la situación económica no les brinda la oportunidad de contar con un abanico más amplio de adquisición.

Adicionalmente de los resultados de las variables, se realizó el análisis de las colonias de procedencia de los encuestados del CUCAH, de lo cual, se obtuvo un registro de 20 colonias de procedencia (Figura 2), las cuales son: Del bosque, Centro, Payo Obispo I, Magisterial, 20 de Noviembre, Adolfo López Mateos, Proterritorio, 5 de Abril, INFONAVIT Santa María, Forjadores, Caribe, Bicentenario, Miraflores, Solidaridad, Fraccionamiento Arboledas I, Pacto Obrero, Jardines, Lagunitas, Las Américas y Calderitas. Este último, es un poblado pequeño, sin embargo, por su proximidad a la zona urbana y gran movilidad poblacional, se le otorgó para efectos de este estudio la categoría de colonia. En suma, son 20 colonias, de un total de 77 colonias y 48 fraccionamientos que integran la ciudad de Chetumal $(\mathrm{H}$. Ayuntamiento de Othón P. Blanco, 2016), indicativo que la 
Figura 2

Ubicación geográficay representatividad poblacional de usuarios-consumidores de las colonias de la ciudad de Chetumal al CUCAH.

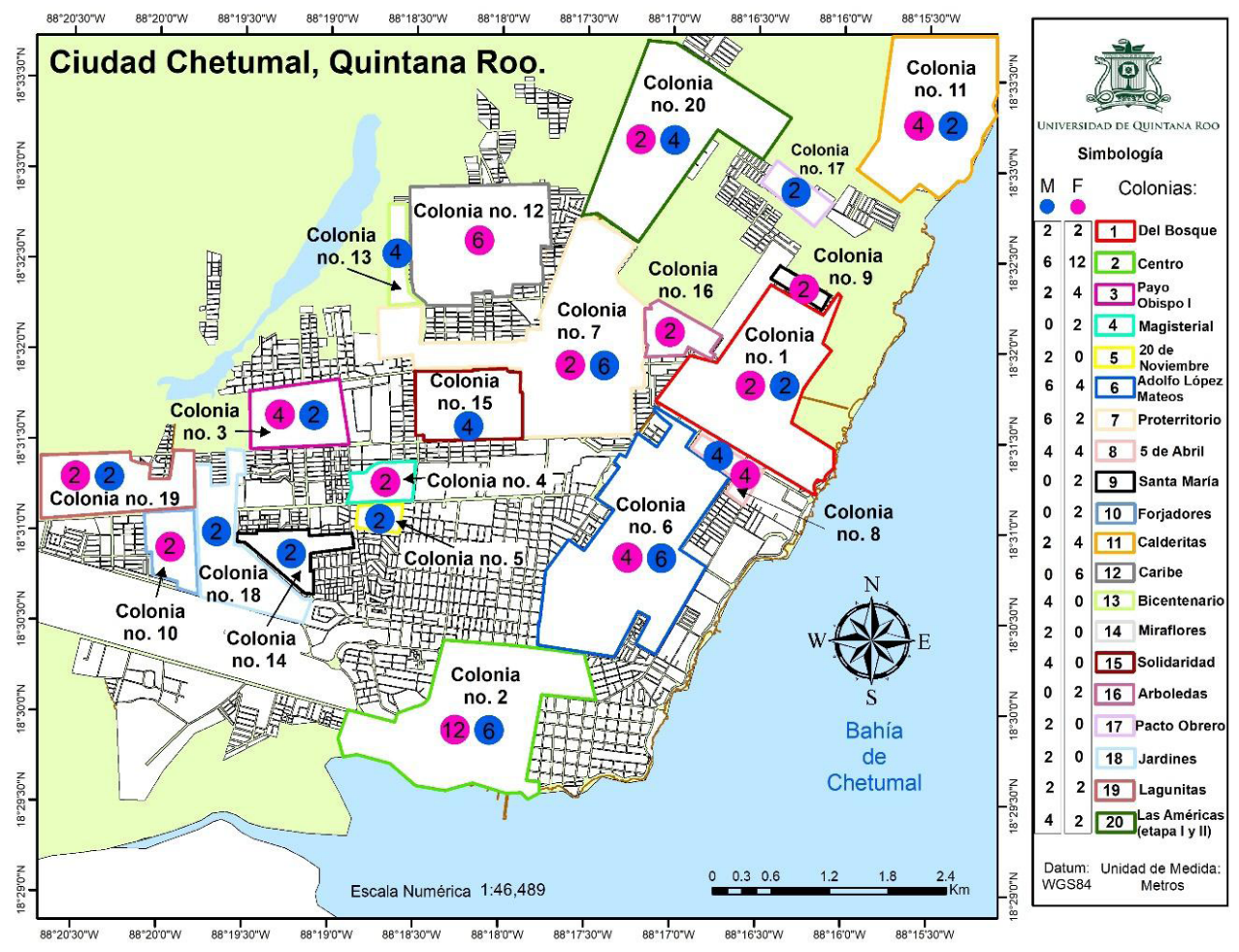

Fuente: Elaboración propia.

representatividad poblacional de procedencia por colonia en la investigación es poco significativa.

Por otra parte, en las colonias identificadas de procedencia generalmente pertenecen a clases sociales media, media baja y baja, no se encuestó a ninguna persona de procedencia de colonia de clase media alta o alta; indicativo, que las personas que van a visitar y consumir al CUCAH son personas de perfil medio a bajo. Se pone en evidencia con estos resultados que los usuarios que llegan al corredor no son representativos de todas las clases sociales que integran la ciudad de Chetumal, motivo por el cual en el indicador de productos deseados encontrados en los establecimientos (visto en el apartado de Acción). El 42\% de los encuestados opinaron con la opción de frecuentemente, entendiéndose que la gran mayoría de los encuestados poseen una capacidad de compra generalmente dirigida a la adquisición de productos de primera y segunda necesidad; para el caso del corredor es destinado a productos de segunda necesidad como lo son el calzado y la ropa.

El hecho que en el corredor urbano comercial no se encontraron ciudadanos de todas las clases sociales, refleja en gran medida la pérdida de preferencia del espacio público como lugar recurrente para visita y consumo. Las tres primeras colonias de procedencia con mayor número de encuestados corresponden en primer lugar a la colonia Centro, con un 18\%; en segundo lugar, se encuentra la colonia Adolfo López Mateos con un $10 \%$ y en tercer lugar la colonia Proterritorio con un $8 \%$ de representatividad. De lo anterior, se observa que los usuarios de mayor representatividad en el CUCAH son los de mayor proximidad hablando en términos espaciales urbanos, correspondientes a colonias con arterias de comunicación directamente conectadas con amplias avenidas, como lo es la Av. Benito Juárez, Av. de los Héroes, Av. Constituyentes del 74 y Calzada Veracruz en sentido norte a sur y que cuentan con transporte urbano colectivo (Figura 2).

\section{Conclusiones}

La aplicación del proceso metodológico en la investigación a partir del análisis empírico del Marco de las Cuatro Aes con el enfoque de Cerdá Suárez et al. (2007), dentro del contexto de Zonas Comerciales Urbanas y/o Centros 
Comerciales Abiertos (CCA), para el caso de estudio del CUCAH, puso de manifiesto desde la perspectiva de los usuarios-consumidores los factores que actualmente inciden en la pérdida del atractivo, así como la revelación de otros elementos que se suman al hecho. Con base a lo anterior, y a manera de variable dependiente evaluada por los usuarios-consumidores, se desprende que el CUCAH ha perdido su atractivo; a pesar de la inversión millonaria aplicada 110 millones de pesos mexicanos en el pasado sexenio del Exgobernador Lic. Roberto Borge Angulo (2011-2016) para posicionarla como el núcleo comercial de la capital del Estado.

Muchos son los factores que inciden en el actual resultado; si bien es cierto, actualmente el componente físicoedificado del corredor urbano comercial fue remodelado y se encuentra en buenas condiciones en términos generales, la remodelación no fue finalizada con todos los alcances planteados en el proyecto ejecutivo, lo cual, hasta el día de hoy continúan sin ser atendidos y concluidos por la actual administración del gobierno estatal. Garantizar el atractivo del corredor, va más allá del componente físico-edificado, tal como se presentó en el caso de estudio, no bastan superficies de rodamiento vehicular y peatonal nuevas con buena iluminación y áreas ajardinadas. Es imprescindible la aplicación de estrategias consideradas como no tangibles, pero de gran importancia de responsabilidad de las gerencias de los establecimientos de los comercios y servicios ubicados a lo largo del corredor urbano comercial, así como el apoyo decidido de la cámara de comercio local que agrupa a la mayoría de los empresarios.

Desde luego, hay factores que son externos al área de estudio y a los propietarios de los comercios, como lo es las rutas de transporte colectivo urbano que permitan la accesibilidad desde cualquier punto de la ciudad hasta el corredor, responsabilidad total del gobierno municipal. Estas debilidades se reflejaron en los resultados del Marco de las Cuatro Aes, en la Accesibilidad sólo 2 indicadores de 4 obtuvieron calificaciones satisfactorias otorgadas por los usuarios-consumidores, las cuales son la buena ubicación y áreas peatonales suficientes del corredor. En cuanto a la dimensión de Atracciones, de los 5 indicadores evaluados sólo 3 fueron evaluados satisfactoriamente por la ciudadanía, los cuales corresponden a buen lugar para comprar, variedad de productos y buen lugar para salir a comer; en lo relativo las Amenidades, de los 4 indicadores encuestados, sólo 1 fue considerado de manera satisfactoria; y para la dimensión de Acción de los 3 indicadores avaluados, sólo 1 fue considerado por los usuarios como satisfactorio.

En resumen, de los 16 indicadores que fueron integrados en el Marco de las Cuatro Aes para el caso de estudio, sólo 7 obtuvieron opiniones positivas por parte de los encuestados, siendo 9 calificados con percepciones negativas; situación por demás preocupante y que viene a reforzar lo referido, el atractivo de un Área Urbana Comercial no se logra o se conserva únicamente implementando estrategias de conservación y/o remodelación del ambiente edificado, los factores humanos, las estrategias del marketing, la buena gestión, coordinación y unidad entre propietarios comerciantes, así como el apoyo y gestión de las cámaras de comercio local para fortalecer a los agremiados, son indispensables para lograr el éxito del atractivo del CUCAH.

Es imperativo que la pasividad de los actores involucrados se transforme en acciones proactivas que permitan incrementar la rentabilidad, competitividad y funcionalidad de la actividad comercial local, así como la calidad del espacio para eficientar las actividades de relaciones y encuentros entre la ciudadanía; de lo contrario, el CUCAH está destinado a ser un espacio desfavorable de todo tipo de atracción para el usuario-consumidor, y con ello el abandono y pérdida de la centralidad del espacio público.

Este estudio fue planteado desde la investigación de la perspectiva del usuario-consumidor por las condiciones extraordinarias en que fue desarrollada (inicio de la pandemia del COVID-19), así como los recursos disponibles. Sin embargo, se contemplan líneas futuras de investigación como la pérdida de atractivo comercial desde la perspectiva de los comerciantes y la conversión del corredor urbano a Centro Comercial Abierto (CCA). Esto último, motivado porque el área de estudio tiene todos los elementos urbanos para tal efecto, desde la perspectiva urbanística y geográfica. 


\section{Referencias bibliográficas}

Aguirre, E. y Fuentes, C. (2013). Escenarios de violencia urbana: usos y percepciones del espacio público relacionado con la vivienda en Ciudad Juárez (1990-2010). En M. Solís, Estudios de lo local en la frontera norte de México. Sujetos, familias, espacios, pp. 125-148. Tijuana: El Colegio de la Frontera Norte.

Arroyo, J. (2018). Espacio público. Entre afirmaciones y desplazamientos. Santa Fe: Universidad Nacional del Litoral.

Bensús, V. (2012). Ideología de la inseguridad y segregación en el espacio público en Lima Metropolitana: el caso de la gestión 2007-2010 en Miraflores. Debates en Sociología, (37), 77-108.

Calderón, E., Giraldo, E. y Jiménez, S. (2018). Imaginarios urbanos y apropiación del espacio público: skatepark del municipio de Rionegro. En C. Arango, Territorio, identidades, comunicación, pp. 77-102. Río Negro: Fondo Editorial Universidad Católica de Oriente.

Calderón, G., Gómez, M., Dávila, L., Osorio, M. y Caro, E. (2020). Habitantes de calle en Medellín, Colombia: sus normas, derechos y deberes. Equidad y Desarrollo, (35), 167-185. https://doi.org/10.19052/eq.vol1.iss35.8

Cao, J., \& Kang, J. (2019). Social relationships and patterns of use in urban public spaces in China and the United Kingdom. Cities, 93, 188-196. https://doi.org/10.1016/j. cities.2019.05.003

Cerdá Suárez, L. M. (2006). Caracterización de los factores de éxito de los centros comerciales abiertos desde la perspectiva de los comerciantes (Tesis doctoral no publicada) Universidad Autónoma de Madrid, Madrid, España.

Cerdá Suárez, L. M. (2016). Dimensiones clave para revitalizar áreas comerciales urbanas: El caso de Getafe, España. Revista de Ciencias Sociales, XXII(1), 98-109.
Cerdá Suárez, L. M., Fernández Nogales, Á., \& Rebollo Arévalo , A. (2007). Atributos para el éxito de una zona comercial: una evidencia empírica en el municipio de Getafe. Distribución y Consumo, 16-41. https://www.mapa. gob.es/ministerio/pags/biblioteca/revistas/pdf_DYC/ DYC_2007_95_16_31.pdf

Flórez Parra, J. M. (2011). Andalucía y los Centros Comerciales Abiertos: El caso de Maracena, Granada. Revista de Estudios Cooperativos, (106), 95-120. https://doi.org/10.5209/ rev_REVE.2011.v106.37374

García-Doménech, S. (2014). Percepción social y estética del espacio urbano en la sociedad contemporánea. Arte, Individuo y Sociedad, 26(2), 301-316. https://doi. org/10.5209/rev_ARIS.2014.v26.n2.41696

García-Domenech, S. (2015). Espacio público y comercio en la ciudad contemporánea. DEARQ - Revista de Arquitectura / Journal of Architecture(17), 29-39. https://www.redalyc. org/pdf/3416/341645612003.pdf

García Gallego, J. M., Chamorro Mera, A. y Palacios González, M. M. (2010). El atractivo de un centro comercial abierto según las opiniones de los clientes y de los empresarios, Aplicación al casco antiguo de Badajoz. Distribución y Consumo, (113), 1-9. https://www.mercasa.es/media/ publicaciones/62/1287435526_1287163463_Art_GALLEGO.pdf

García, C. y Ruíz, O. (2011). La segregación territorial y el rezago en el sur de la Ciudad de Mérida, como el resultado del crecimiento urbano descontrolado. Quivera, 13(1), 122-138. https://quivera.uaemex.mx/article/view/10161

Gasca Zamora, J. (2017). Centros comerciales de la Ciudad de México: el ascenso de los negocios inmobiliarios orientados al consumo. EURE (Santiago), 43(170), 7396. http://dx.doi.org/10.4067/s0250-71612017000300073

H. Ayuntamiento de Ohtón P. Blanco. (2016). Plan Municipal de Desarrollo 2016-2018. Chetumal. Recuperado el 23 de marzo de 2020 de http://www.opb.gob.mx/portal/ wp-content/uploads/2016/07/Plan-Municipal-deDesarrollo-2016-2018.pdf 
H. Ayuntamiento de Othón P. Blanco. (2018). Programa de Desarrollo Urbano de Chetumal-Calderitas-Subteniente LópezHuay-Pix y Xul-Há. Municipio de Othón P. Blanco, Estado de Quintana Roo. http://www.opb.gob.mx/portal/ wp-content/uploads/transparencia/93/I/f/PDU2018/ PDU\%20integrado\%2019012018-publicacion\%20digital.pdf

Instituto Nacional de Estadística y Geografía. (2010). Principales resultados del Censo de Población y Vivienda 2010 Quintana Roo. Aguascalientes, México.http://coespo.qroo.gob.mx/ Descargas/doc/23_principales_resultados_Censo $\% 20$ de\%20Población $\% 20 Y \% 20$ Vivienda\%202010\%20QROO.pdf

Iso Tinoco, A. (2013). El espacio público en los centros comerciales a cielo abierto, el caso de Pamplona. Zainak, (36), 213-230. https://www.researchgate.net/ publication/283072830_El_espacio_publico_en_los_centros_ comerciales_a_cielo_abierto_El_caso_de_Pamplona

Janoschka, M. (2016). Gentrificación, desplazamiento, desposesión: Procesos urbanos claves en América Latina. Revista INVI, 31(88), 27-71. http://dx.doi.org/10.4067/ S0718-83582016000300002

Jasso López, L. C. (2015). ¿Por qué la gente se siente insegura en el espacio público?, La política pública de prevención situacional de delito. (Tesis de Doctorado). Doctorado en Políticas Públicas, Centro de Investigación y docencia económicas,A.C. México. https://cide.repositorioinstitucional. mx/jspui/bitstream/1011/684/1/000145898_documento.pdf

López, J., López, L. y Carvajal, M. (2020). ¿Equidad en la dotación de espacio público en Bogotá?. Revista de Arquitectura (Bogotá), 22(1), 18-29. https://dx.doi. org/10.14718/revarq.2020.2606

Márquez Ulin, J. (10 de Septiembre de 2016). El Gobernador Inaugura Obras en la Av. de de los Héroes. Diario de Quintana Roo. https://dqr.com.mx/sections/othon-pblanco/35474-nueva-y-funcional-imagen.html

Moravcová, J., Jiri, P., Pekna, D., Moravcova, V., \& Novakova, N. (2020). The Role of Public Spaces in Small Municipality. IntechOpen. https://doi.org/10.5772/intechopen.89881
Pancorbo Sandoval, J. A., Benavides, S. y León Sánchez, D. (2011). Centro comercial abierto, una estrategia para la revitalización del Centro Histórico Urbano de Matanzas. Arquitectura y Urbanismo, XXXII(1), 46-49. http://www. redalyc.org/articulo.oa?id=376839861005

Pascual-González, A. y Peña-Díaz, A. (2012). Espacios abiertos de uso público. AU. Arquitectura y Urbanismo, XXXIII(1), 25-42. http://scielo.sld.cu/pdf/au/v33n1/au030112.pdf

Pérez H., E. (2004). Percepción del espacio público. Bitácora Urbano Territorial, 1(8), 27-31. https://revistas.unal.edu. co/index.php/bitacora/article/view/18753/19648

Poder Ejecutivo, Secretaría de Hacienda y Credito Público. (2013). Presupuesto de Egresos de la Federación para el Ejercicio Fiscal 2014. México: Diario Oficial de la Federación.

Ramírez Kurí, P. (2016). La reinvención del espacio público en la ciudad fragmentada. México: Universidad Nacional Autónoma de México.

S.I.C.I. DOMINUS.SL. (2007). Análisis de la Estructura del Centro Comercial de Motril. Granada: Mesones. http:// www.observatorioempleomotril.es/fileadmin/estudios/ comercio/009_ANALISIS_DE_LA_ESTRUCTURA_DEL_ CENTRO_COMERCIAL_ABIERTO_D.pdf

Segovia, O. (2007). Espacios Públicos y Construcción Scoial, Hacia un Ejercicio de Ciudadanía. Santiago de Chile: Ediciones SUR.

Vecslir, L. y Rodríguez, L. (2018). Centros Comerciales a Cielo Abierto como política de renovación de las centralidades tradicionales en el Conurbano Bonaerense. Territorios, (38), 15-40. http://dx.doi.org/10.12804/revistas.urosario. edu.co/territorios/a.5499

Xacur Maiza, J. Á. (5 de abril de 2017). Apuntes para una historia de la Zona Libre ¿Una economía fuerte y estable? La Jornada Maya (447), p. 8.https://issuu.com/lajornadamaya/ docs/la_jornada_maya____mie__rcoles_5_de 